\title{
Feature Selection and Binarization for On-Line Signature Recognition
}

\author{
Emanuele Maiorana, Patrizio Campisi, and Alessandro Neri \\ Dipartimento di Elettronica Applicata, Università degli Studi "Roma Tre", \\ Via della Vasca Navale 84, 00146 Roma, Italy \\ \{maiorana, campisi, neri\}@uniroma3.it
}

\begin{abstract}
The representation of a biometric trait through a set of parametric features is commonly employed in many biometric authentication systems. In order to avoid any loss of useful information, large sets of features have been defined for biometric characteristics such as signature, gait or face. However, the proposed sets often contain features which are irrelevant, correlated with other features, or even unreliable. In this paper we propose two different approaches for the selection of those features which guarantee the best recognition performances. Moreover, we also face the problem of the binary representation of the selected features. Specifically, an algorithm which selects the minimum number of bits which should be assigned to a given feature, in order to not affect the recognition performances, is here proposed. The effectiveness of the proposed approaches is tested considering a watermarking based on-line signature authentication system, and employing the public MCYT online signature corpus as experimental database.
\end{abstract}

Keywords: Biometrics, On-line Signature, Feature Selection, Feature Binarization, Watermarking.

\section{Introduction}

Loosely speaking, biometric systems are essentially pattern recognition applications, which perform user authentication by analyzing people physiological or behavioral characteristics. As in the classical framework of a pattern recognition system, a generic biometric system can be represented as a cascade of four main modules performing data acquisition, feature extraction, template storing and template matching [1. Specifically, the feature extraction module processes the acquired biometric data, namely the pattern, to extract a set of discriminatory features, taken as representative of the acquired data.

Two types of features can be distinguished: parameters and functions. When parameters are used, the considered biometrics is characterized by a vector of elements, each representative of a feature. When functions are used, the given biometrics is characterized in terms of a set of time or space dependent sequences.

However, the sets of parametric features proposed in literature often contain features which are irrelevant or correlated with other features, or even unreliable. This typically happens because many redundant features are included 
in the employed sets, in order to minimize the chances to loose useful information. It is worth pointing out that the representation of biometric data in high-dimensional feature spaces involves several issues: by considering features with high intra-user variability and low inter-user variability, the performance of the employed classification method can be negatively affected, thus resulting in sub-optimal recognition performances 2. Moreover, the use of a large number of features usually increases the processing and the matching time. High dimensional data also impose severe requirements for storage space and communication bandwidth, which are crucial factors in the actual deployment of a real-time system. Therefore, the selection of the most discriminative parametric features is an issue of paramount importance for many biometric systems. Specifically, the feature selection process holds a significant relevance when considering behavioral biometrics, due to the high intra-class variability which can be experienced by extracting features from different acquisitions.

An algorithm for the selection of those features which offer the best representation of a speech sample has been proposed in [3], where genetic algorithms are employed in conjunction with feed forward neural networks. An approach combining genetic algorithms and particle swarm optimization has been proposed in 4 for the selection of relevant keystroke dynamics features. Moreover, mutual information is exploited in [5] for human gait feature selection. As far as signature recognition is concerned, the problem of feature selection has been discussed in [6], where the selection of a unique set of features for each individual has been suggested, and in [7, where a modified version of the Fisher ratio has been employed as cost function for the selection of local and global signature parametric features. In [8] a statistical distance, based on the difference between the mean values of different users' features, has been defined. In [9], a cost function based on the Equal Error Rate (EER) has been employed to select a subset of signature features, while a consistency measure has been introduced in [10] to rank signature features according to their importance. An algorithm employed to rank a set of on-line signature parametric features has been also presented in [11, while the use of genetic algorithms has been proposed in [12] to select an optimal set of on-line signature features.

In this contribution, a feature selection procedure, based on the distance between distributions of genuine and forgers matching scores, is introduced. Moreover, an approach which allows to binarize a feature set, using only the bits needed to not degrade the system performance, is proposed. The proposed feature selection and binarization procedures are then applied to signature based authentication using watermarking techniques proposed by the authors in [13], as an application example.

\section{Feature Selection}

In this Section, the proposed procedures for the selection of the most representative features belonging to a given set are detailed. Specifically, the presented approaches perform feature selection on the basis of the distances between genuine and forgers matching scores distributions. In fact, it is well known from 
pattern classification that a properly defined feature selection stage should be designed using the matcher employed for the classification. Therefore, the presented approaches can be applied for the selection of the best parametric features extracted from any biometrics. Instead of defining different sets of features for different users as proposed in [6], the proposed approaches define a unique set of features which has to be employed for each enrolled user.

In the proposed approach we assume that a training set of biometric acquisitions, taken from $U$ different users, is available and can be employed to analyze the contribution of each feature to the recognition process. Specifically, we assume that, for each user $u, T$ genuine biometric samples are acquired, and that $J$ forgery samples are also available. These latter samples can be either biometric acquisitions taken from different users, or skilled forgeries which mimick the biometrics of the considered user. Moreover, we assume that the matching module is based on the computation of the Mahalanobis distance between feature vectors extracted from different biometric samples.

Specifically, $K$ features are extracted, for each user, from each of $E$ genuine samples, selected among the $T$ samples available in the training data set. The mean and the standard deviation vectors $\boldsymbol{\mu}^{u}$ and $\boldsymbol{\sigma}^{u}$, of the $K$ features are then computed. The feature vectors $\mathbf{g}_{i}^{u}$, with $i=1, \ldots, I$, where $I=T-E$ represents the remaining genuine acquisitions, are evaluated. The feature vector extracted from the $j$-th forged sample, with $j=1, \ldots, J$, of the user $u$ is indicated as $\mathbf{f}_{j}^{u}$.

An approach to determine a feature ranking, and an iterative approach to determine the best feature to add to an already given set, are described in Sections 2.1 and 2.2, respectively.

\subsection{Features Selection: Ranking Approach}

The features selection approach here proposed assigns a reliability measure to each feature, which can then be ranked accordingly: the features with the $L$ highest reliability values are then selected to efficiently represent the considered biometric. Specifically, given a feature $k$, the following distances are computed for each user $u$ belonging to the training set, $u=1,2, \ldots, U$ :

$$
\mathbf{G}^{u}[i, k]=\frac{\left|\mathbf{g}_{i}^{u}[k]-\boldsymbol{\mu}^{u}[k]\right|}{\boldsymbol{\sigma}^{u}[k]} ; \quad \mathbf{F}^{u}[j, k]=\frac{\left|\mathbf{f}_{j}^{u}[k]-\boldsymbol{\mu}^{u}[k]\right|}{\boldsymbol{\sigma}^{u}[k]}
$$

and collected in the matrices $\mathbf{G}^{u}$ and $\mathbf{F}^{u}$ of dimensions $I \times K$ and $J \times K$ respectively, with $u=1,2, \ldots, U$.

Then, for each user $u$ the distance $\boldsymbol{\vartheta}^{u}[k]$, for the $k$-th feature, between $\mathbf{G}^{u}[i, k]$ and $\mathbf{F}^{u}[j, k]$ is evaluated, employing one of the distances defined in Section [2.3. The reliability of the $k$-th feature is then computed by taking the median value $\boldsymbol{\lambda}[k]$ among the $U$ values $\left\{\boldsymbol{\vartheta}^{u}[k]\right\}$. The features with the $L$ highest values of $\boldsymbol{\lambda}[k]$ are considered to be the most representative ones for recognition purposes.

\subsection{Features Selection: Incremental Approach}

The approach described in Section 2.1 analyzes the reliability of each considered feature, without taking into account the correlation between different features. 
However, in most cases the considered features are correlated. Therefore the selection of the best feature which can be added to a given set should be performed on the basis of the already selected ones. In order to take into account possible dependencies between the considered features, in this paper we present an incremental approach.

Specifically, if a subset comprising the $L$ most reliable features, out of the available $K$, has to be identified, the proposed procedure has to be run by iterating $L$ times the algorithm detailed in the following.

Let us define with $\mathcal{L}_{l}$ the set of the selected features at step $l$, with $l=$ $1, \cdots, L$ and $\mathcal{L}_{0}$ defines the empty set at the initialization step. Let $\boldsymbol{\Delta} G_{0}^{u}[i, k]$ and $\Delta F_{0}^{u}[j, k]$ the zero matrices of dimensions $I \times K$ and $J \times K$ respectively.

The generic $l$-th step of the proposed algorithm is defined as follows:

1. for each user $u$, with $u=1,2, \cdots, U$, computation of $\mathbf{G}_{l}^{u}[i, k]$ and $\mathbf{F}_{l}^{u}[j, k]$, for $k \in \mathcal{K} \backslash \mathcal{L}_{l-1}$, where "\" is the difference operator, as:

$$
\begin{aligned}
& \mathbf{G}_{l}^{u}[i, k]=\sqrt{\left(\frac{\mathbf{g}_{i}^{u}[k]-\boldsymbol{\mu}^{u}[k]}{\boldsymbol{\sigma}^{u}[k]}\right)^{2}+\Delta G_{l-1}^{u}[i, k]}, i=1, \ldots, I ; \\
& \mathbf{F}_{l}^{u}[j, k]=\sqrt{\left(\frac{\mathbf{f}_{j}^{u}[k]-\boldsymbol{\mu}^{u}[k]}{\boldsymbol{\sigma}^{u}[k]}\right)^{2}+\boldsymbol{\Delta} F_{l-1}^{u}[j, k]}, j=1, \ldots, J ;
\end{aligned}
$$

2. for each user $u$, with $u=1,2, \cdots, U$, computation of the distances $\boldsymbol{\vartheta}_{l}^{u}[k]$ between $\mathbf{G}_{l}^{u}[i, k]$ and $\mathbf{F}_{l}^{u}[j, k]$ for $k \in \mathcal{K} \backslash \mathcal{L}_{l-1}$. The measures detailed in Section 2.3 are employed to evaluate the required distances;

3. for each feature $k \in \mathcal{K} \backslash \mathcal{L}_{l-1}$, evaluation of the median values $\boldsymbol{\lambda}_{l}[k]$ of the $U$ values $\left\{\boldsymbol{\vartheta}_{l}^{u}[k]\right\}$;

4. selection of the feature $\tilde{k} \in \mathcal{K} \backslash \mathcal{L}_{l-1}$ which possesses the highest reliability value $\boldsymbol{\lambda}_{l}[\tilde{k}]$;

5. update the set of selected features as $\mathcal{L}_{l}=\mathcal{L}_{l-1} \bigcup \tilde{k}$;

6. for each user $u$, with $u=1,2, \cdots, U$, update the matrices $\Delta G_{l}^{u}$ and $\Delta F_{l}^{u}$ as follows:

$$
\begin{aligned}
& \boldsymbol{\Delta} G_{l}^{u}[i, k]=\boldsymbol{\Delta} G_{l-1}^{u}[i, k]+\left(\frac{\mathbf{g}_{i}^{u}[\tilde{k}]-\boldsymbol{\mu}^{u}[\tilde{k}]}{\boldsymbol{\sigma}^{u}[\tilde{k}]}\right)^{2} \cdot \mathbf{o}[k] \\
& \boldsymbol{\Delta} F_{l}^{u}[j, k]=\boldsymbol{\Delta} F_{l-1}^{u}[j, k]+\left(\frac{\mathbf{f}_{j}^{u}[\tilde{k}]-\boldsymbol{\mu}^{u}[\tilde{k}]}{\boldsymbol{\sigma}^{u}[\tilde{k}]}\right)^{2} \cdot \mathbf{o}[k]
\end{aligned}
$$

being $\mathbf{o}[k]$ a row vector with only ones, and "." the column-by-row multiplication operator, for every $k \in \mathcal{K} \backslash \mathcal{L}_{l}$.

7. if $l<L$ then $l=l+1$, otherwise the iterations stop.

\subsection{Distribution Distances}

As already pointed out, both the proposed procedures for feature selection compute a distance between two distributions. In the proposed implementations, two different measures have been employed to evaluate the distance between the probability mass function $P_{X}$ and $P_{Y}$ of the score sets $X$ and $Y$, respectively. 
EER Based Distance. The distance $\Delta_{E E R}\left(P_{X}, P_{Y}\right)$ is defined using the EER. Specifically, being $P_{X}$ the score probability mass function obtained from the matching of authentic biometric samples, and $P_{Y}$ the score probability mass function obtained from the matching of forgeries, the resulting EER is then computed. We then set $\Delta_{E E R}\left(P_{X}, P_{Y}\right)=1-E E R$.

Kullback-Leibler Based Distance. The distance $\Delta_{K L}\left(P_{X} \| P_{Y}\right)$ is based on the estimation of the score probability mass function $P_{X}$, obtained from the matching of authentic biometric samples, and the score probability mass function $P_{Y}$, obtained from the matching of forgeries. The Kullback-Leibler (KL) divergence between the two estimated densities is eventually computed. Specifically, the Parzen window estimator [15] with a Gaussian kernel is employed to estimate $P_{X}$ and $P_{Y}$. The distance $D_{K L}\left(P_{X} \| P_{Y}\right)$ is then obtained as

$$
D_{K L}\left(P_{X} \| P_{Y}\right)=\sum_{i} P_{X}(i) \ln \frac{P_{X}(i)}{P_{Y}(i)} .
$$

In Section [5] it shown that the proposed feature selection approaches lead to recognition rates, expressed in terms of EER, better than those obtained using the approach in [11].

\section{Features Binarization}

In this Section, the method employed to determine the minimum number of bits necessary to represent a given feature, without affecting the achievable recognition performances, is detailed. As for the feature selection algorithms described in Section 2 it is assumed that a training set of biometric acquisitions can be analyzed. Using the notations introduced in Section 2, we first estimate the minimum and maximum value for a given feature $k$, with $k=1,2, \cdots, K$.

Specifically, the maximum and minimum allowable score values, $\mathbf{M}[k]$ and $\mathbf{m}[k]$, for the $k$-th feature are computed as follow. The $\alpha \%$ highest score values are discarded thus obtaining as maximum allowable value for the $k$-th feature $\boldsymbol{\zeta}[k]$. Then, the maximum allowable score value, $\mathbf{M}[k]$ is set as $\mathbf{M}[k]=\boldsymbol{\zeta}[k]+$ $\eta|\boldsymbol{\zeta}[k]|$, where $\eta>0$. Similarly, the minimum allowable score value $\mathbf{m}[k]$ for the $k$-th features is computed as $\mathbf{m}[k]=\boldsymbol{\varsigma}[k]-\eta|\boldsymbol{\varsigma}[k]|$, being $\boldsymbol{\varsigma}[k]$ the minimum score value obtained by discarding the $\alpha \%$ lowest scores. The parameters $\alpha$ and $\eta$ are selected in order to limit the effects of possible outliers in the estimation of the maximum and minimum values of the given distribution. Once the allowed range for the score of the $k$-th feature has been determined, the following procedure is employed to verify whether a given number of bits $b$ can be used to represent the mean value of the $k$-th feature, without affecting the achievable verification performances.

For each user $u$, the matrices $\mathbf{G}^{u}$ and $\mathbf{F}^{u}$, whose values are given in (1) are evaluated. The values $\boldsymbol{\mu}^{u}[k]$ are then binarized employing $b$ bits for each element, taking into account that the $k$-th feature assumes values into the interval $[\mathbf{m}[k], \mathbf{M}[k]]$. The decoded version of the binarized $k$-th feature mean vector is indicated as $\boldsymbol{\nu}^{u}[k]$, and employed to determine the distances: 


$$
\mathbf{G b}^{u}[i, k]=\frac{\left|\mathbf{g}_{i}^{u}[k]-\boldsymbol{\nu}^{u}[k]\right|}{\boldsymbol{\sigma}^{u}[k]} ; \quad \mathbf{F b}^{u}[j, k]=\frac{\left|\mathbf{f}_{j}^{u}[k]-\boldsymbol{\nu}^{u}[k]\right|}{\boldsymbol{\sigma}^{u}[k]} .
$$

For each user $u$, a measure representing the goodness of the employed binarization is then computed as:

$$
\gamma_{b}^{u}[k]=\frac{1}{I} \sum_{i=1}^{I}\left(\mathbf{G}^{u}[i, k]-\mathbf{G b}^{u}[i, k]\right)-\frac{1}{J} \sum_{j=1}^{J}\left(\mathbf{F}^{u}[j, k]-\mathbf{F b}^{u}[j, k]\right) .
$$

In fact, it can be observed that when the term $\frac{1}{I} \sum_{i=1}^{I}\left(\mathbf{G}^{u}[i, k]-\mathbf{G b}^{u}[i, k]\right)$ is positive, the binarization of the $k$-th feature results in an improvement of the recognition performances. Similarly, an improvement of the recognition accuracy can be obtained when the term $\frac{1}{J} \sum_{j=1}^{f}\left(\mathbf{F}^{u}[j, k]-\mathbf{F b}^{u}[j, k]\right)$ is negative. Therefore, once the terms $\gamma_{b}^{u}[k]$ have been computed for each available user $u$, a quality measure for the binarization of the $k$-th feature with $b$ bits can be obtained as $\boldsymbol{\Gamma}_{b}[k]=\sum_{u=1}^{U} \boldsymbol{\gamma}_{b}^{u}[k] / U$. If $\boldsymbol{\Gamma}_{b}[k]<0$, the binarization of the mean values of feature $k$ with $b$ bits, for each enrolled user, will result in a performance degradation. When $\boldsymbol{\Gamma}_{b}[k] \simeq 0$ the recognition performances are not affected by the binarization, and could be even improved if $\boldsymbol{\Gamma}_{b}[k]>0$. The minimum number of bits which should be employed to binarize the $k$-th feature is therefore obtained as the lowest value $b$ for which steadily results $\left|\boldsymbol{\Gamma}_{b}[k]\right|<\epsilon$, where $\varepsilon$ is a predefined threshold, with $\varepsilon \simeq 0$.

\section{Feature Selection and Binarization: An Application to Signature Watermarking}

The proposed approaches for feature selection and binarization have been applied to the system presented in 13 , which is here briefly summarized.

In [13] a multi-level signature based authentication system, where watermarking is employed to hide a set of dynamic signature features into an image of the signature itself, has been described. Specifically, during the enrollment stage $E$ signatures are acquired for each user through an electronic pad, and some relevant dynamic features are extracted from them. The selected features are then embedded into the signature images, by means of watermarking techniques based on the Radon transform. The images are represented using signatures' pressure values, being thus grey-scale images instead of simply binary ones. Among the enrolled signature of a given user, the one whose static features are the closest to the estimated mean is selected to host the chosen dynamic features.

The Malahanobis distance is used to compare both dynamic and static features in the authentication stage. The employed authentication system allows to obtain two levels of security, according to the considered application: when a low security level is needed, the authentication can be performed using only some selected static features, extracted from the signature images. When a higher level of security is required, authentication is accomplished using the embedded dynamic features, by themselves or together with the selected static features. 


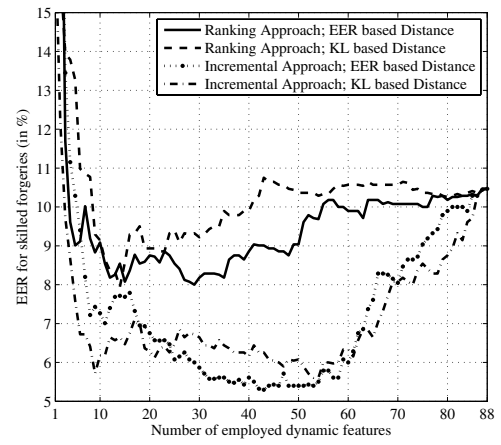

(a)

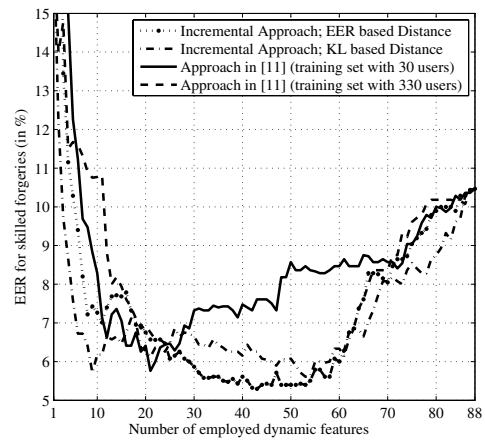

(b)

Fig. 1. Application of the proposed feature selection approaches to dynamic signature features. (a): Comparison between the approaches in Section [2.1] and 2.2. (b): Comparison between the approaches in Section 2.2 and in 11$]$.

In this latter case, score fusion techniques [16] can be employed to combine the information extracted from static and dynamic signature features.

As explained in [13, the considered on-line signature based authentication system requires a set of dynamic features to be selected, and also a binarization procedure of the chosen features. Specifically, in order to provide resilience against errors during the mark extraction phase, the binary strings which are embedded into the signature images have to be protected with an error correcting code (ECC). It is therefore important to generate the binary marks employing as more dynamic features as possible, while containing the final dimension of the binary string to be encoded by the employed ECC, and later embedded in the considered signature image. In 13 eleven dynamic features are selected for the embedding, and binarized using a non-optimal procedure, leading to 92 bits. Then, the $\mathrm{BCH}$ code $(127,92)$ is employed to provide an error correction capability of 5 bits. In Section 5 it is shown that, employing the proposed binarization approach, 17 dynamic features can be represented using a binary string of 92 bits, thus leading to an optimal bit allocation, which allows to increase the number of the embedded features.

\section{Experimental Results}

An extensive set of experimental tests has been performed using the public MCYT on-line signature corpus [14, comprising 100 users, for each of which 25 genuine signatures and 25 skilled forgeries have been captured during five different sessions. The employed database has been divided in two disjoint data sets: a training set, which comprises the genuine and forged signatures of the first 30 users, and a test set, which includes the genuine and forged signatures of the remaining 70 users. The training set has been employed to select the best dynamic and static features, and also to determine the number of bits which has 


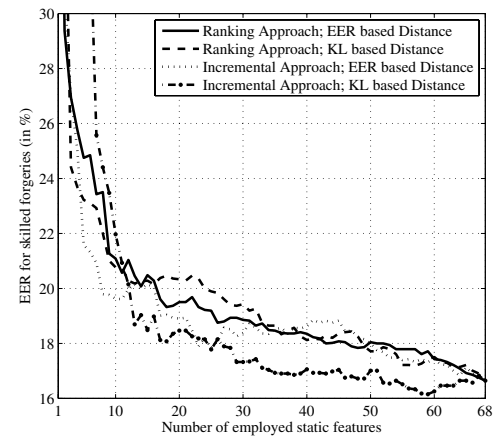

(a)

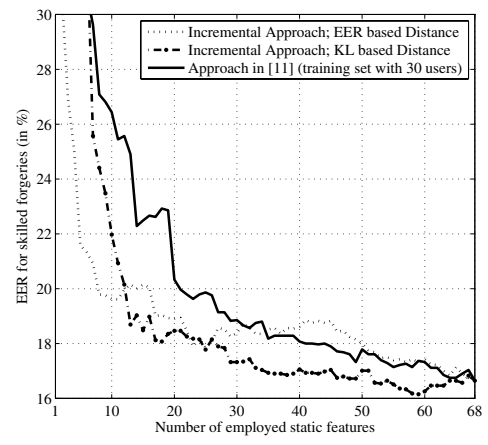

(b)

Fig. 2. Application of the proposed feature selection approaches to static signature features. (a): Comparison between the approaches in Section 2.1 and 2.2 (b): Comparison between the approaches in Section 2.2 and in 11 .

to be assigned for the binarization of each selected dynamic feature. Specifically, the feature vectors $\mathbf{f}_{j}^{u}, j=1, \ldots, 25$ and $u=1, \ldots, 30$, employed in the proposed methods of Section 2, are extracted from the skilled forgeries available in the training set. The effectiveness of the proposed methods has been tested:

- by evaluating the recognition rates in terms of EER, and by comparing them with those obtained employing the method described in [11;

- by applying the proposed feature selection and binarization approaches to the watermarking based system described in [13].

We have tested the proposed feature selection algorithms employing a set of 88 dynamic features, derived from the set presented in [1] by discarding the parameters related to static information. The results obtained when taking $E=10$ signatures for the enrollment phase are shown in Figure 1. Specifically, Figure 1(a) shows the comparison between the methods described in Section 2.1] and 2.2. employing both the distances defined in Section 2.3. The results are displayed in terms of the EER, evaluated over the test set with 70 users and considering skilled forgeries, versus the number of employed features. The obtained results illustrate that the incremental procedure of Section 2.2 performs better than the ranking based one. This means that the employed features are highly correlated, being then not possible to perform efficiently the selection of a feature regardless of the already selected ones. Figure1(b) shows the comparison between the incremental approach presented in Section 2.3 and the feature selection method presented in [11. Specifically, this latter is applied by performing the feature selection procedure over the training set with $U=30$ users, and then evaluating the recognition performances over the test set. It can be seen that the proposed incremental methods performs better than the one in 11. Moreover, the proposed approach is also compared with the feature ranking presented in [11, which has been estimated employing the whole MCYT database with 330 user as training set. The incremental procedure described in Section 2.2 still 


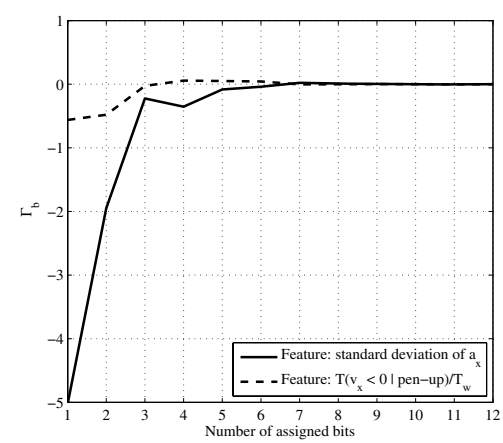

(a)

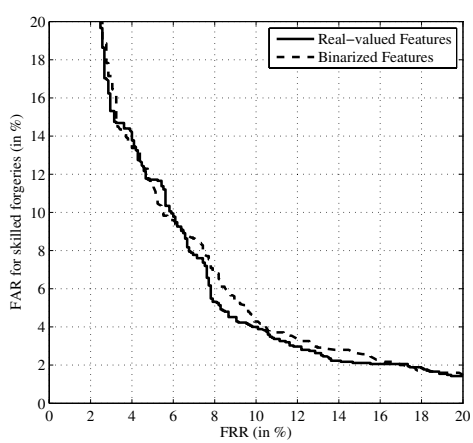

(b)

Fig. 3. Analysis of the proposed feature binarization approach. (a): Quality measure $\boldsymbol{\Gamma}_{b}$ with respect of the number of employed bits, for two considered dynamic features; (b): Effectiveness of the proposed binarization method, verified over the test data set.

performs better when tested for low values of selected features, and allows to reach the same lowest EER which can be achieved employing the feature ranking presented in [11, that is equal to $5.4 \%$. The same comparisons between different feature selection methods have also been carried out considering the set of 68 static features employed in [13. The obtained results are illustrated in Figure 2. which still validates the effectiveness of the proposed approaches.

As for the second experiment, the feature selection and binarization approaches proposed in this paper are applied to the watermarking based system briefly outlined in Section 4. Specifically, we have selected 17 dynamics features out of the considered 88 ones, employing the proposed incremental feature selection approach with the distribution distance based on the KL divergence. The feature binarization approach described in Section 3 has been employed to represent the 17 selected dynamics features with only 92 bits. The necessary bitdepths have been estimated over the training set with 30 users. The employed parameters are $\alpha=0.01$ and $\eta=0.2$. The behavior of the quality measure $\boldsymbol{\Gamma}_{b}$ introduced in Section 3 is illustrated in Figure 3(a). According to what has been explained in Section 3 , the feature "standard deviation of $a_{x}$ " (defined in [11]) can be binarized employing 7 bits, while feature " $T\left(v_{x}<0 \mid\right.$ pen-up) $/ T_{w}$ " (defined in [11]) can be represented employing only 4 bits. The effectiveness of the proposed binarization approach has been verified by analyzing the recognition performances computed over the test data set, while employing 17 dynamics features. Specifically, the performances obtained employing real-valued vectors $\boldsymbol{\mu}^{u}$ to represent the intra-class mean are compared in Figure 3(b) with those achievable employing vectors $\boldsymbol{\nu}^{u}$, generated from the binary representation of the considered dynamic features. It is evident that the recognition performances, expressed in terms of False Recognition Rate (FRR) and False Acceptance Rate (FAR) for skilled forgeries, are not affected by the proposed binarization, even if the employed 17 dynamic features are represented with 92 bits, whereas the same amount of bits were employed in 13 to represent only 11 dynamic features. 


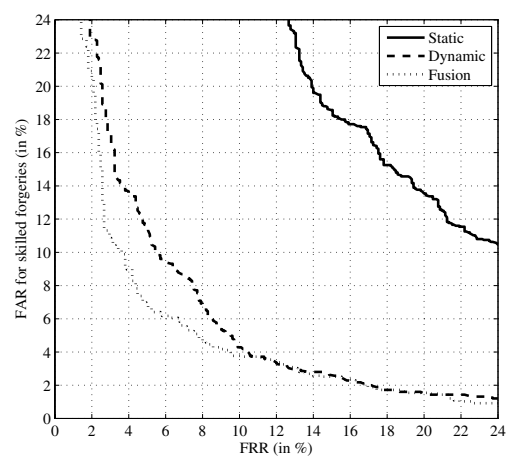

Fig. 4. Performances of the proposed on-line based signature authentication system

The recognition performances achievable for the considered multi-level signature based authentication system of Section 4 are displayed in Figure 4 . The proposed incremental feature selection with the KL distribution distance is employed to select the best 50 static features. The achieved EER for static features, computed over the test data set, is equal to $17.07 \%$. The EER achievable employing 17 dynamic features, selected according to the proposed incremental approach, is equal to $7.41 \%$. Combining both static and dynamic features through a min-max normalization technique [16, together with a sum rule, results in a EER of $6.16 \%$. The parameters needed for the implementation of the min-max normalization technique are estimated using the training data set, and later applied to fuse the scores obtained over the test data set.

\section{References}

1. Jain, A.K., Ross, A., Prabhakar, S.: An Introduction to Biometric Recognition. IEEE Trans. on Circuits and Systems for Video Technology 14(1) (2004)

2. Hughes, G.: On the mean accuracy of statistical pattern recognizers. IEEE Transactions on Information Theory 14(1), 55-63 (1968)

3. Quixtiano-Xicohtencatl, R., Flores-Pulido, L., Reyes-Galaviz, O.F.: Feature Selection for a Fast Speaker Detection System with Neural Networks and Genetic Algorithms. In: 15th International Conference on Computing (CIC) (2006)

4. Azevedo, G.L.F., Cavalcanti, G.D.C., Filho, E.C.B.: An approach to feature selection for keystroke dynamics systems based on PSO and feature weighting. In: IEEE Congress on Evolutionary Computation (CEC), pp. 3577-3584 (2007)

5. Baofeng, G., Nixon, M.S.: Gait Feature Subset Selection by Mutual Information. In: IEEE Conference on Biometrics: Theory, Applications, and Systems (BTAS) (2007)

6. Brittan, P., Fairhurst, M.C.: Feature selection in automatic signature verification. In: IEE Colloquium on Image Processing for Biometric Measurement (1994)

7. Richiardi, J., Hamed, K., Drygajlo, A.: Local and global feature selection for online signature verification. In: Conference on Document Analysis and Recognition $(2005)$ 
8. Lee, L., Berger, T., Aviczer, E.: Reliable on-line human signature verification systems. IEEE Trans. on PAMI 18(6), 643-647 (1996)

9. Crane, H., Ostrem, J.: Automatic signature verification using a three-axis forcesensitive pen. IEEE Trans. on Systems, Man, and Cybernetics (1983)

10. Lei, H., Govindaraju, V.: A study on the consistency of features for on-line signature verification. In: IAPR SSPR (2004)

11. Fiérrez-Aguilar, J., Nanni, L., Lopez-Peñalba, J., Ortega-Garcia, J., Maltoni, D.: An on-line signature verification system based on fusion of local and global information. In: Kanade, T., Jain, A., Ratha, N.K. (eds.) AVBPA 2005. LNCS, vol. 3546, pp. 523-532. Springer, Heidelberg (2005)

12. Galbally, J., Fierrez, J., Freire, M.R., Ortega-Garcia, J.: Feature Selection Based on Genetic Algorithms for On-Line Signature Verification. In: IEEE AIAT (2007)

13. Maiorana, E., Campisi, P., Neri, A.: Biometric Signature Authentication using Radon Transform-based Watermarking techniques. In: IEEE BSYM (2007)

14. Ortega-Garcia, J., et al.: MCYT baseline corpus: A bimodal biometric database. IEE Proceedings Vision, Image and Signal Processing 150(6) (2003)

15. Parzen, E.: On Estimation of a Probability Density Function and Mode. Annals of Mathematical Statistics 33, 1065-1076 (1962)

16. Ross, A., Nandakumar, K., Jain, A.K.: Handbook of Multibiometrics. Springer, Heidelberg (2006) 